\title{
A Molecular Dynamics Method to Identify the Liquidus and Solidus in a Binary Phase Diagram
}

\author{
C. O. T. Galvin ${ }^{\mathrm{a}, \mathrm{b}}$, R. W. Grimes ${ }^{\mathrm{b}}$, P. A. Burr ${ }^{\mathrm{a}, *}$ \\ ${ }^{a}$ School of Mechanical and Manufacturing Engineering, University of New South Wales, Kensington, 2052, NSW, Australia \\ ${ }^{b}$ Department of Materials, Imperial College London, London, SW7 2AZ, UK
}

\begin{abstract}
A method is presented adopting the phase coexistence technique within molecular dynamics simulations to identify the liquidus and solidus of binary systems. The Compositional Moving Interface method is applied to the case study of the $\mathrm{Cu}-\mathrm{Ni}$ system and compared against a thermodynamic end-point model where the input parameters are determined using the same MD potential. This is a simple and powerful method to predict the solidus and liquidus boundary of a binary phase diagram for mixed systems calculated from the dynamics of a simulation.
\end{abstract}

The melting temperature of simple (unary) systems can be calculated using computational techniques based on molecular dynamics (MD) simulations, such as the Z-method [1-4] and the moving interface (or phase coexistence) method [5-9]. However, binary systems will not have one melting point, but a two-phase region bound by a liquidus and a solidus. When those methods are applied to a binary mixture a "pseudounary" melting point is found [10-12], but it is not clear whether these melting points correspond to the solidus, the liquidus or somewhere in between. On the other hand, current methods to predict solidus and liquid of binary systems from atomic scale simulations rely on assumptions of ideal mixing to extrapolate the results from the end members.

A previous attempt in the literature to predict the phase boundaries from two-phase MD simulation proved successful on oxygen-carbon-selenium plasma mixtures [13]. However, the method is cumbersome, sensitive to a choice of local order parameter, and computationally intensive compared to the proposed method. Further, work by Coura et al. [14] use MD to study directional growth of binary mixtures by investigating an equilibrium crystal-fluid interface. Here a variant of the moving interface method is presented that can predict the liquidus and solidus boundaries and thereby construct a binary phase diagram. We call this method the compositional moving interface or CMI.

The proposed CMI method has been developed to predict the melting behaviour of solutions that do not follow ideal laws of mixing. It has been previously used, along with other approaches, to show deviation from ideal behavior in $\mathrm{U}$, Th and $\mathrm{Pu}$ oxide systems [15]. However, it is not possible to test the validity of the

*Corresponding author p.burr@unsw.edu.au

Preprint submitted to Computational Materials Science

August 25, 2020 
approach against systems that deviate strongly from ideal mixing as there are no computational predictions for such systems that can be validated to a high degree of certainty. Direct comparison with experiments for strongly non-ideal systems, on the other hand, is hindered by the additional uncertainties introduced by the quality and transferability of the empirical potentials used. Instead, here we test the validity of the proposed method against ideal mixing laws, in a self-consistent manner. More specifically, we use the $\mathrm{Cu}-\mathrm{Ni}$ system as a case study, as it is a well studied system and for which reliable empirical potentials exist. $\mathrm{Cu}-\mathrm{Ni}$ is an isomorphous system that displays complete solid state miscibility at high temperatures, with small enthalpy of mixing [16-22], and where the radii differ only slightly. Under these conditions, mixing behaviour is well approximated by that of a regular or ideal solution. Therefore, the predictions of liquidus and solidus made assuming ideal mixing, and informed by parameters obtained though MD simulations, are directly comparable with the CMI predictions made using the same empirical potentials and simulations parameters.

MD simulations were conducted using the Large-scale Atomic/Molecular Massively Parallel Simulator (LAMMPS) [23]. The study employed the embedded atom potential model by Sheng et al. [24],1. For all simulations a Nosé-Hoover barostat with a relaxation time of 0.5 ps was used to control the pressure while the system temperature was controlled with a Nosé-Hoove thermostat and a relaxation time of $0.1 \mathrm{ps.} \mathrm{An}$ MD timestep of $1 \mathrm{fs}$ was used in all calculations unless otherwise stated.

First the melting temperature $\left(T_{m}\right)$ of the end members were calculated using the conventional moving interface method. The moving interface simulation cell is constructed by joining two equilibrated simulation cells, one constituting a solid phase and the other a liquid phase both at the same temperature and conventionally of the same composition. Depending on the simulation temperature, the interface between the two phases will move. If the system energy is too low to maintain the liquid phase the interface will move into the liquid and the system will progressively solidify. Likewise, if the system energy is sufficiently high the solid will melt into the liquid. In the current study, the temperature range in which neither phase grows by an appreciable amount (up to three atomic planes) in $1 \mathrm{~ns}$, is only $5 \mathrm{~K}$.

The simulation cell was constructed as follows. A $10 \times 10 \times 60$ cell, containing 24,000 atoms, was heated to a target temperature at $0 \mathrm{GPa}$ and allowed to equilibrate for $20 \mathrm{ps}$. After this, the cell was split into two adjacent regions: the solid region, in which the atoms' positions were frozen in place (i.e. no integration of trajectories), and the liquid region, which was heated to $2500 \mathrm{~K}$ (above the melting points of $\mathrm{Cu}$ and $\mathrm{Ni}$ ) over $20 \mathrm{ps}$, held at that temperature for another 20 ps to establish a true liquid distribution, and cooled back down to the test temperature. During this process the system pressure was relieved by applying a barostat in the z-direction only (the direction normal to the interface). Following this, the atoms in the solid region were unfrozen and using NPT dynamics with the barostat acting in all directions the whole simulation cell

\footnotetext{
${ }^{1}$ The tabulated version of this potential can be found at https://sites.google.com/site/eampotentials/Home/CuNi
} 
was equilibrated at the target temperature for $1 \mathrm{~ns}$.

Using the moving interface approach described above, the $T_{m}$ of $\mathrm{Cu}$ and $\mathrm{Ni}$ were calculated as $1385 \pm 2.5 \mathrm{~K}$ for $\mathrm{Cu}$ and $1760 \pm 2.5 \mathrm{~K}$ for $\mathrm{Ni}$, respectively. The error associated with the MD melting points are determined as the range in which melting occurs (i.e. the minimum and maximum refer to a simulation that did not and did melt, respectively). These temperatures are in close agreement with experimental $T_{m}$ observations of $1358 \mathrm{~K}$ and $1728 \mathrm{~K}$ [25]. A small difference between MD and experimental melting temperatures is expected, and a difference of only $\sim 30 \mathrm{~K}$ provides confidence in the potentials. The predicted enthalpy of fusion values ${ }^{2}, \Delta H_{m}$, at those temperatures are $12.23 \mathrm{~kJ} \mathrm{~mol}^{-1}$ and $17.64 \mathrm{~kJ} \mathrm{~mol}^{-1}$ for $\mathrm{Cu}$ and $\mathrm{Ni}$ respectively, also in close agreement with experiment [26] $\left(12.93 \mathrm{~kJ} \mathrm{~mol}^{-1}\right.$ and $\left.17.04 \mathrm{~kJ} \mathrm{~mol}^{-1}\right)$.

For an ideal solution (i.e. one that has zero enthalpy of mixing and zero volume change on mixing) the liquidus and solidus can be calculated from their heats of fusion and melting temperatures with the following relations [27], where it has been assumed that the heat capacity difference between the liquid and solid phases is zero.

$$
\begin{gathered}
\ln \frac{x_{s}^{A}}{x_{l}^{A}}=\frac{\Delta H_{m}^{A}}{R}\left(\frac{1}{T_{m}^{A}}-\frac{1}{T}\right) \\
\ln \frac{1-x_{s}^{A}}{1-x_{l}^{A}}=\frac{\Delta H_{m}^{B}}{R}\left(\frac{1}{T_{m}^{B}}-\frac{1}{T}\right)
\end{gathered}
$$

Here $R$ is the ideal gas constant, $T_{m}^{i}$ is the melting temperatures of the end-member $i(A$ or $B), \Delta H_{m}^{i}$ is its enthalpy of fusion and $x_{s}^{A}$ and $x_{l}^{A}$ are the mole fractions in the solidus and liquidus for end-member $A$ at temperature $T$.

The proposed CMI method, while computationally similar to the conventional moving interface method, is different because the liquid and solid regions have different compositions. For each CMI calculation two $12 \times 12 \times 24$ supercells of different compositions were joined to create a $12 \times 12 \times 48$ supercell. Firstly, the whole system was equilibrated to the target temperature at $0 \mathrm{GPa}$, then the CMI method was implemented, as described below, for a duration of 1 ns. A calculation of a liquidus point using the CMI method is summarised below with respect to the schematic $\mathrm{Cu}-\mathrm{Ni}$ binary phase diagram in figure 1 .

1. An initial test temperature $T_{i}$ is chosen (black dashed line in figure 1), typically between the melting point of the end members; in this range, the end member $A$ is known to be in the liquid state while the end member $B$ is known to be solid.

2. The liquid phase is created with composition of the end-member $A$ and equilibrated at temperature $T_{i}$ (the black point in figure 1).

3. A test composition $x_{i}$ of the $A-B$ mixture is chosen (pink dashed line in figure 1), and the solid phase is created with this composition and equilibrated at the test temperature $T_{i}$.

\footnotetext{
${ }^{2} \mathrm{~A} 32,000$ atom supercell was heated and cooled with $\Delta T=25 \mathrm{~K}$. The system was equilibrated for 100 ps and average values obtained from the final $20 \mathrm{ps}$.
} 


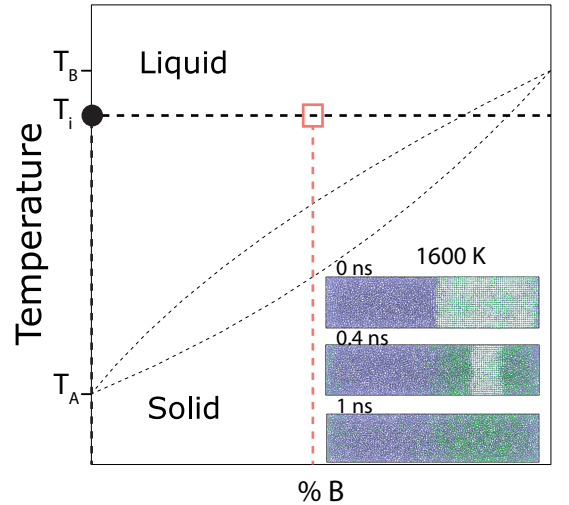

(a)

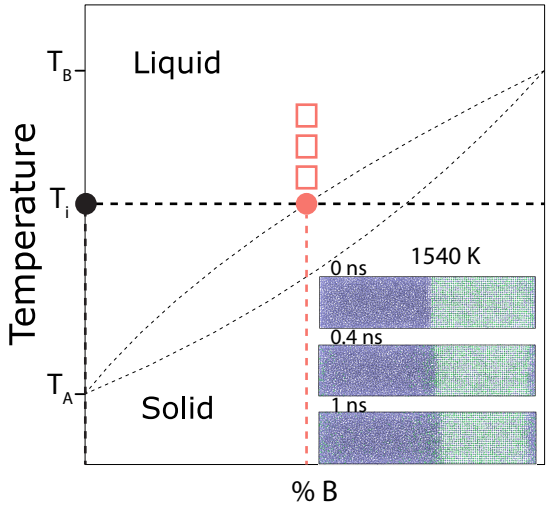

(b)

Figure 1: Schematic of the Cu-Ni binary phase diagram where $T_{A}$ is the melting point of $A$ and $T_{B}$ is the melting point of $B$. The CMI method is outlined for two simulations. The filled black circle indicates the liquid composition at temperature $T_{i}$, shown in blue in the three blocks on the right-hand side. The open pink square identifies the composition of the (initially) solid, shown in green in the three blocks. (a) represents a temperature above the liquidus such that the solid region melts at the chosen composition. (b) shows a temperature/composition where both the liquid and solid phase can coexist and is marked by a filled pink circle signifying the liquidus has been reached.

4. The two structures are joined to form a single simulation supercell with the two distinct compositions and two liquid/solid interfaces. A simulation cell for this case is shown in figure 1 a at 0 ns (i.e. before equilibration of the whole system, $t=0)$.

5. Running an MD simulation at $T_{i}$ in figure 1a, the liquid side of the system will consume the solid so that the whole system will melt at this system energy. This process is presented for the $\mathrm{Cu}_{50} \mathrm{Ni}_{50}$ system at $1610 \mathrm{~K}$ in figure 1a. This case is represented by an open pink square as it has been determined that the whole system melts, that is, it is above the liquidus.

6. The MD calculation procedure is repeated for lower temperatures. In figure $1 \mathrm{~b}$ the next two lower temperatures are still above the liquidus so are therefore also represented by open pink squares.

7. The temperature is lowered until it is determined that the system has crossed the liquidus line. At this point the system energy is such that both the solid and liquid regimes coexist. This point is represented by a solid pink circle in figure 1b. Evidence that the system is now in the two phase regime is presented in figure $1 \mathrm{~b}$ as a series of three simulation snapshots, starting at 0 ns and ending at $1 \mathrm{~ns}$.

To calculate a point on the solidus the converse process is carried out. That is, a series of simulations are carried out with progressively increasing temperature for a system in which the composition of the solid phase is the end-member $B$ and the composition of the liquid phase is the target $A$ - $B$ solution. The whole system will solidify until a temperature where both the solid and liquid regimes exist, that is, the system has crossed the solidus. It is important to understand that the melting process, at the atomic scale, is not an equilibrium process. The liquid is to be a medium into which the solid melts and therefore it is not crucial 


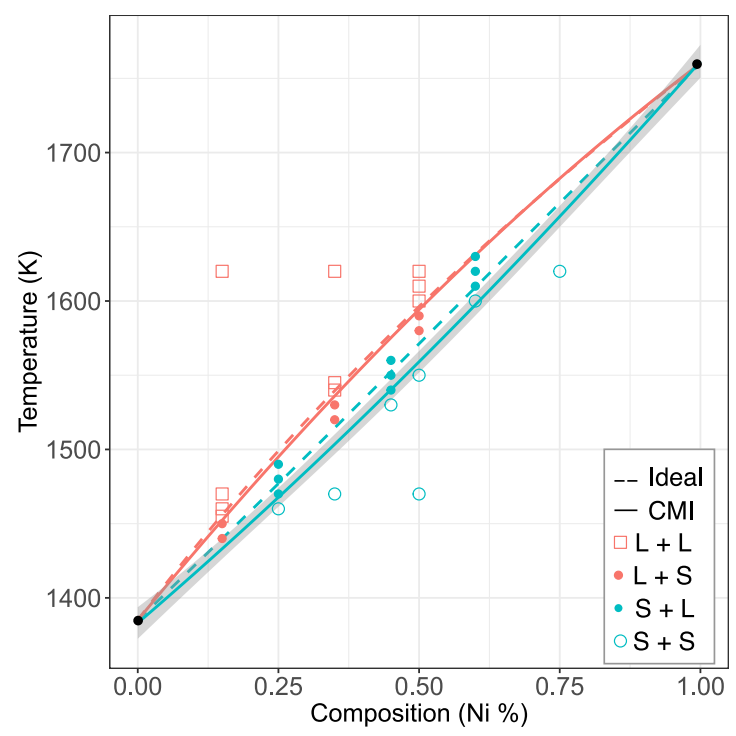

Figure 2: Binary phase diagram predictions from the ideal model (dashed lines) and from CMI MD calculations (solid lines). The grey shaded region represents the $75 \%$ confidence interval of the quadratic fit. Pink and blue symbols represent liquidus and solidus calculations, respectively. Open and filled symbols represent simulations that ended in a single phase and two-phase equilibrium respectively.

that the liquid has the equilibrium composition. Of course, the liquid can not deviate too far from the equilibrium composition, but as long as it is close, a concentration gradient will form in the liquid near the interface during the first few timesteps of the simulation. This local change in composition at the interface is what enables our simplified approach to calculate the liquidus and solidus.

Figure 2 shows the compendium of $\mathrm{CMI}$ predictions for the $\mathrm{Cu}-\mathrm{Ni}$ phase diagram and its comparison with the ideal model. The pink and blue dashed lines are the ideal liquidus and solidus lines determined via equations 1 and 2 using the calculated melting points and enthalpies of fusion. Using the CMI method, the open pink squares and open blue circles indicate when the MD supercell completely melts or solidifies, indicating the system is in the liquid or solid region of the phase diagram. The solid pink circles represent when the system melts but there is some solid left. The solid blue circles represent when some of the liquid solidifies but there is some liquid left. Using the MD results, a $2^{\text {nd }}$ order polynomial was fit to the temperature identifying the limiting two-phase temperatures at specific concentrations that therefore outline the liquidus and solidus; represented by the solid pink and blue curves respectively. This fit was chosen as a visual aid to observe the liquidus and solidus on the binary phase diagram. The grey shaded region is a $75 \%$ confidence level (confidence interval of liquidus is narrower than line thickness and therefore not visible).

It is found that the MD simulations are able to generate data of sufficient temperature resolution to be used to generate liquidus and solidus boundaries that correspond closely with the ideal predictions for the $\mathrm{Cu}-\mathrm{Ni}$ system [25]. The calculated liquidus from the CMI method is almost identical to the ideal case whereas the solidus is slightly lower than the ideal case. Since these boundaries are predicted from dynamics 


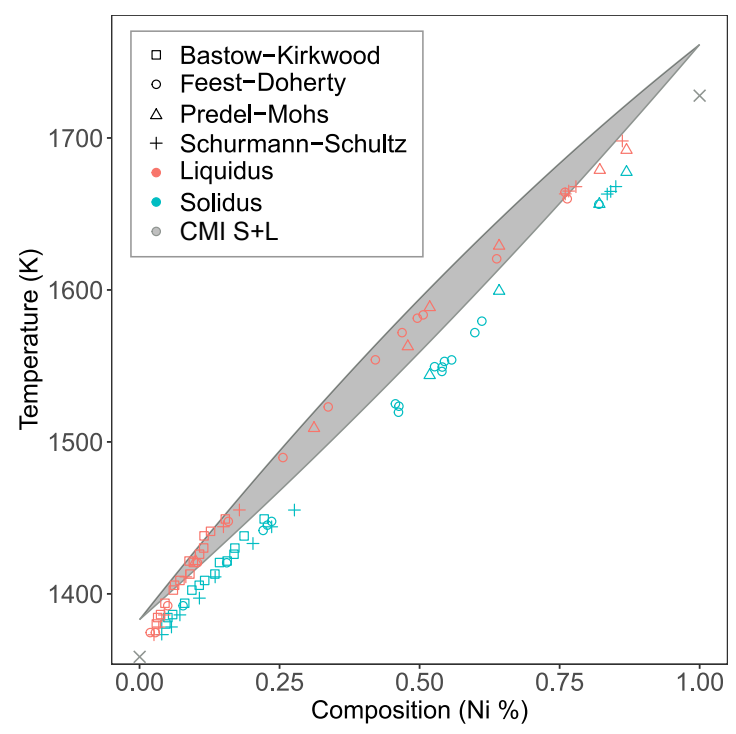

Figure 3: Blue and pink points represent experimental values [28-31] for the Cu-Ni binary phase diagram solidus and liquidus, respectively. The grey shaded region represents the solid-liquid region predicted by the CMI method.

it is possible that they are closer to "reality" than assuming the ideal case. The discrepancy between CMI predictions and those using ideal mixing laws are too small to be corroborated by the experimental data [25]. Direct correspondence to the phase diagram is also made difficult because the melting points of the endmembers are different to those predicted by the potentials used here. Therefore, the two sets of the liquidus and solidus lines are displaced from each other. Nevertheless, the predicted and experimental temperature separations between liquidus and solidus lines are close, as shown in figure 3.

Interestingly, for the liquidus simulations, a small amount of loss of solid phase is always observed, even when the temperature is below the liquidus boundary. This is explained by heterogeneous melting at the interface; a process that may occur at temperatures much lower than the melting point, but over significantly longer time-scales. Interface heterogeneous melting is driven by the concentration gradient of $B$ atoms (Ni in this case) into the liquid phase (nominally pure $A$, or $\mathrm{Cu}$ ). In a dynamic equilibrium, atoms at the interface continually melt and solidify. However, as the $B$ atoms $(\mathrm{Ni})$ melt into the liquid $A$ phase $(\mathrm{Cu})$, they will form a concentration gradient that drives diffusion of $B$ atoms towards the bulk of the liquid phase. As a consequence, the interface will recede into the solid phase.

To test the sensitivity of this process, and more generally to ascertain the effect of approximating the composition of the liquid as end-member $A$, a number of simulations were repeated where the solid had a composition of $\mathrm{Cu}_{50} \mathrm{Ni}_{50}$ and the pure $\mathrm{Cu}$ liquid side was replaced by increasing amounts of $\mathrm{Ni}$, from $5 \%$ to $50 \%$ with steps of $5 \%$. Two temperatures were chosen for these simulation runs, $1560 \mathrm{~K}$ (in the solid region) and $1580 \mathrm{~K}$ (in the 2-phase region). At $1580 \mathrm{~K}$, all the simulation runs yield the same result: a system that contains a liquid phase with some solid, consistent with being in the 2-phase region. A general 
trend was observed whereby as the $\mathrm{Ni}$ concentration increases, the rate at which the solid melts decreases. This suggests that, on the time-scale of these calculations, the liquid region provides a low Ni concentration medium that facilitates the formation of an appropriate liquid structure with which the solid can form a stable interface. At $1560 \mathrm{~K}$, from Ni 0\% to 40\% there is slight melting and the system consists of liquid and solid. When the $\mathrm{Ni}$ concentration is increased to $45 \%$ at this temperature, the system beings to solidify, however over the time-scale of our simulation there is still liquid in the system. Finally, when the Ni content is at $50 \%$ the system fully solidifies. This strong sensitivity to the liquid concentration past $40 \% \mathrm{Ni}$, when the solid contains $50 \% \mathrm{Ni}$, is a further indication that at $1560 \mathrm{~K}$ we are close to the solidus.

This may be a consequence of the melting being liquid diffusion rate limited, rather than heat transfer limited. Thus, the liquid region does not have to be pure $A$, its purpose is to provide a low $B$ concentration medium into which the solid can melt. Equally, for the solidus calculation; for this system on these timescales, the solid does not have to be pure $B$, its purpose is to provide an appropriate surface onto which solid can precipitate.

The results obtained from the $\mathrm{Cu}-\mathrm{Ni}$ test case are encouraging, and it is proposed that this CMI model may be used more generally to predict the behaviour of more complex types of solid solutions that deviate strongly from ideal mixing behaviour.

\section{Acknowledgements}

This work was undertaken with the assistance of resources and services from the National Computational Infrastructure (NCI), which is supported by the Australian Government, and the Multi-modal Australian ScienceS Imaging and Visualisation Environment (MASSIVE). This research was also supported by resources provided by the Pawsey Supercomputing Centre with funding from the Australian Government and the Government of Western Australia. Additional computational resources were provided by Intersect Australia Ltd.

\section{Data Availability}

The data that support the findings of this study are available from doi: 10.17632/xydncc3cy7.1.

\section{References}

\section{References}

[1] S. Davis, A. Belonoshko, A. Rosengren, A. Duin, and B. Johansson. Molecular dynamics simulation of zirconia melting. Open Physics, 8(5):789-797, jan 2010.

[2] A. R. Finney and P. M. Rodger. Applying the $\mathrm{Z}$ method to estimate temperatures of melting in structure II clathrate hydrates. Physical Chemistry Chemical Physics, 13(44):19979, sep 2011.

[3] S. Wang, G. Zhang, H. Liu, and H. Song. Modified Z method to calculate melting curve by molecular dynamics. The Journal of Chemical Physics, 138(13):134101, apr 2013

[4] F. González-Cataldo, S. Davis, and G. Gutiérrez. Z method calculations to determine the melting curve of silica at high pressures. Journal of Physics: Conference Series, 720(1):012032, may 2016. 
[5] J. R. Morris, C. Z. Wang, K. M. Ho, and C. T. Chan. Melting line of aluminum from simulations of coexisting phases. Physical Review B, 49(5):3109-3115, feb 1994.

[6] A. B. Belonoshko, R. Ahuja, O. Eriksson, and B. Johansson. Quasi ab initio molecular dynamic study of Cu melting. Physical Review B, 61(6):3838-3844, feb 2000.

[7] J. R. Morris and X. Song. The melting lines of model systems calculated from coexistence simulations. The Journal of Chemical Physics, 116(21):9352-9358, jun 2002.

[8] J. J. Hoyt, Mark Asta, and Alain Karma. Atomistic simulation methods for computing the kinetic coefficient in solid-liquid systems. Interface Science, 10(2-3):181-189, 2002.

[9] K. Govers, S. Lemehov, M. Hou, and M. Verwerft. Comparison of interatomic potentials for UO2. Journal of Nuclear Materials, 376(1):66-77, may 2008.

[10] M. W. D. Cooper, M. J. D. Rushton, and R. W. Grimes. A many-body potential approach to modelling the thermomechanical properties of actinide oxides. Journal of Physics: Condensed Matter, 26(10):105401, mar 2014.

[11] P. S. Ghosh, N. Kuganathan, C. O. T. Galvin, A. Arya, G. K. Dey, B. K. Dutta, and R. W. Grimes. Melting behavior of (Th,U)O 2 and (Th,Pu)O 2 mixed oxides. Journal of Nuclear Materials, 479:112-122, oct 2016.

[12] D. G. Frost, C. O. T. Galvin, M. W. D. Cooper, E. G. Obbard and P. A. Burr. Thermophysical Properties of UraniaZirconia (U,Zr)O2 Mixed Oxides By Molecular Dynamics. Journal of Nuclear Materials, 151876, jan 2020.

[13] A. S. Schneider, J. Hughto, C. J. Horowitz, and D. K. Berry. Direct molecular dynamics simulation of liquid-solid phase equilibria for two-component plasmas. Physical Review E, 85(6):066405, jun 2012.

[14] P. Z. Coura, O. N. Mesquita, and B. V. Costa. Molecular-dynamics simulation of directional growth of binary mixtures. Physical Review B, 59(5):3408-3413, feb 1999.

[15] C.O.T. Galvin, P.A. Burr, M.W.D. Cooper, P.C.M. Fossati, and R.W. Grimes. Using molecular dynamics to predict the solidus and liquidus of mixed oxides $(\mathrm{Th}, \mathrm{U}) \mathrm{O}_{2},(\mathrm{Th}, \mathrm{Pu}) \mathrm{O}_{2}$ and $(\mathrm{Pu}, \mathrm{U}) \mathrm{O}_{2}$. Journal of Nuclear Materials, 534:152127, jun 2020 .

[16] S. M. Foiles. Calculation of the surface segregation of Ni-Cu alloys with the use of the embedded-atom method. Physical Review B, 32(12):7685-7693, dec 1985.

[17] S. M. Foiles, M. I. Baskes, and M. S. Daw. Embedded-atom-method functions for the fcc metals Cu, Ag, Au, Ni, Pd, Pt, and their alloys. Physical Review B, 33(12):7983-7991, jun 1986.

[18] S. Srikanth and K. T. Jacob. Thermodynamic properties of $\mathrm{Cu}-\mathrm{Ni}$ alloys: measurements and assessment. Materials Science and Technology, 5(5):427-434, may 1989.

[19] S. an Mey. Thermodynamic re-evaluation of the Cu-Ni system. Calphad, 16(3):255-260, jul 1992.

[20] G. Bonny, R. C. Pasianot, N. Castin, and L. Malerba. Ternary $\mathrm{Fe}-\mathrm{Cu}-\mathrm{Ni}$ many-body potential to model reactor pressure vessel steels: First validation by simulated thermal annealing. Philosophical Magazine, 89(34-36):3531-3546, dec 2009.

[21] B. Onat and S. Durukanoğlu. An optimized interatomic potential for $\mathrm{Cu}-\mathrm{Ni}$ alloys with the embedded-atom method. Journal of Physics: Condensed Matter, 26(3):035404, jan 2014.

[22] A. Manzoor, S. Pandey, D. Chakraborty, S. R. Phillpot, and D. S Aidhy. Entropy contributions to phase stability in binary random solid solutions. npj Computational Materials, 4(1):47, dec 2018.

[23] S. Plimpton. Fast Parallel Algorithms for Short-Range Molecular Dynamics, 1995.

[24] H. W. Sheng, M. J. Kramer, A. Cadien, T. Fujita, and M. W. Chen. Highly optimized embedded-atom-method potentials for fourteen fcc metals. Physical Review B, 83(13):134118, apr 2011.

[25] M. A. Turchanin and P. G. Agraval. Phase equilibria and thermodynamics of binary copper systems with 3d-metals. VI. Copper-nickel system. Powder Metallurgy and Metal Ceramics, 46(9-10):467-477, sep 2007.

[26] D. W. H. Rankin. CRC handbook of chemistry and physics, 89th edition, edited by David R. Lide. Crystallography Reviews, 15(3):223-224, jul 2009.

[27] C. D. Thurmond. Equilibrium Thermochemistry of Solid and Liquid Alloys of Germanium and of Silicon. I. The Solubility of Ge and Si in Elements of Groups III, IV and V. The Journal of Physical Chemistry, 57(8):827-830, aug 1953.

[28] B. D. Bastow and D. H. Kirkwood Solid/liquid equilibrium in the copper-nickel-tin system determined by microprobe analysis. J. Inst. Metals, 99(9):277-283, 1971.

[29] E. A. Feest and R. D. Doherty The Cu-Ni equilibrium phase diagram. J. Inst. Metals, 3:102-103, 1971.

[30] B. Predel and R. Mohs. Thermodynamische Untersuchung flüssiger Nickel-Kupfer-Legierungen. Archiv für das Eisenhüttenwesen, 42(8):575-579, aug 1971.

[31] E. Schurmann and E. Schultz Untersuchengen zum Verlauf der Liquidus und Solidus linien in den Systemen KupferMangan und Kupfer-Nickel. Z. Metallkd., 62(10):758-762, 1971. 